\title{
Construction of a Penrose Diagram for a Spatially Coherent Evaporating Black Hole
}

\author{
Beth A. Brown \\ James Lindesayt \\ Computational Physics Laboratory \\ Howard University, Washington, D.C. 20059
}

\begin{abstract}
A Penrose diagram is constructed for an example black hole that evaporates at a steady rate as measured by a distant observer, until the mass vanishes, yielding a final state Minkowski space-time. Coordinate dependencies of significant features, such as the horizon and coordinate anomalies, are clearly demonstrated on the diagram. The large-scale causal structure of the space-time is briefly discussed.
\end{abstract}

\section{Introduction}

Often, one's intuitive feel for the behavior of a dynamic black hole is guided by the assumption that the quasi-static dynamics are dominated by small modifications on the geometry of the related static black hole. However, there is some evidence[1] that the asymptotic behaviors of dynamic geometries differ from those of static geometries. Indeed, one expects that the initiation and final evaporation processes of black hole dynamics should involve spatial coherence on scales comparable to those describing the medium scale structure of the space-time. It is therefore of interest to examine examples of temporally dynamic geometries, along with their correspondence to static space-times.

The non-orthogonal temporal coordinate associated with the river model of black holes 22 and related descriptions of dynamic horizons [3] has been

\footnotetext{
*e-mail address, beth.a.brown@nasa.gov

$\dagger$ e-mail address, jlslac@slac.stanford.edu
} 
shown to provide a convenient parameter for describing the evolution of a black hole without introducing new physical singularities due to coordinate anomalies away from the point of symmetry $r=0$. Such a time coordinate mixes sufficiently with the radial spatial coordinate in a manner that gives some spatial coherence to the "nearby" dynamics described using this temporal parameter. One expects that any coherent processes associated with the radiations of evaporating black holes will have spatial scales comparable to the radial mass scale (e.g. Schwarzschild radius) of the geometry. The initiation, growth by accretion, evaporation, and finality of a dynamic black hole should have aspects of spatial coherence on the scales associated with those local "microscopic" dynamics.

This paper develops the Penrose diagram of a geometry with a steady rate of evaporation as introduced in a previous paper[1] in order to examine the large-scale causal structure of an example dynamic black hole. The particular example is chosen because classical and quantum solutions can be obtained in a straightforward manner.

\section{Geometry of an Evaporating Black Hole}

\subsection{Form of the metric}

For the present examination, the space-time metric will take the form

$$
d s^{2}=-\left(1-\frac{R_{M}\left(c t_{a}\right)}{r}\right)\left(d c t_{a}\right)^{2}+2 \sqrt{\frac{R_{M}\left(c t_{a}\right)}{r}} d c t_{a} d r+d r^{2}+r^{2} d \omega^{2}
$$

where $d \omega^{2} \equiv d \theta^{2}+\sin ^{2} \theta d \phi^{2}$. This space-time asymptotically corresponds with Minkowski space $\left(c t_{a}, r, \theta, \phi\right)$ in a manner similar to (but not necessarily identical to) the behavior of a Schwarzschild geometry. Therefore, $c t_{a}$ represents the time coordinate of an asymptotic observer. As demonstrated in reference [1] the Ricci scalar

$$
\mathcal{R}=\frac{3 \dot{R}_{M}}{2 r^{2}} \sqrt{\frac{r}{R_{M}}}
$$

is non-singular away from a physical singularity at the origin, and vanishes

for a static radial mass scale $\dot{R}_{M} \rightarrow 0$. This means that the invariant curvature (and any related invariant physical parameter) is nowhere singular 
away from the origin. Any coordinate singularities manifest only in components unique to that particular coordinate representation, and do not represent singularities in the physical content of the space-time.

\subsection{Evolution of the horizon}

The radial null surfaces for the metric specified in Eq. 2.1 satisfy

$$
\frac{d r_{\gamma}}{d c t_{a}}=-\sqrt{\frac{R_{M}}{r_{\gamma}}} \pm 1
$$

Outgoing photons (where $r_{\gamma}$ increases with $c t_{a}$ ) traverse trajectories that correspond to the upper sign. The radial coordinate corresponding to the horizon is given by the particular null surface proportional to the radial mass scale $R_{H}=\frac{R_{M}}{\zeta_{H}}$, where

$$
\frac{d R_{H}}{d c t_{a}}=-\sqrt{\frac{R_{M}}{R_{H}}}+1 \quad, \quad \dot{R}_{M}=\zeta_{H}\left(1-\sqrt{\zeta_{H}}\right) \quad, \quad R_{H}=\frac{R_{M}}{\left(1-\dot{R}_{H}\right)^{2}} \text {. }
$$

For the case presently being examined, the horizon is seen to be always within the radial mass scale $R_{H}<R_{M}$.

\subsection{Diagonalization of the metric form}

The construction of orthogonal temporal-radial coordinates presents a more direct intuitive representation for the characterization of physical dynamics. If one attempts a coordinate transformation of the form

$$
d c t_{a}=A\left(c t_{a}, r\right) d c t_{D}+\Delta\left(c t_{a}, r\right) d r \quad, \quad d r=d r_{D}
$$

the function $\Delta$ can be chosen to algebraically diagonalize the metric in Eq. 2.1 if it is of the form

$$
\Delta\left(c t_{a}, r\right)=\frac{\sqrt{\frac{R_{M}\left(c t_{a}\right)}{r}}}{1-\frac{R_{M}\left(c t_{a}\right)}{r}} .
$$

Observers using coordinates $\left(c t_{D}, r, \theta, \phi\right)$, including the diagonal temporal coordinate $c t_{D}$, describe no frame-dragging affects in this geometry. The 
diagonalized metric then takes the form

$d s^{2}=-\left(1-\frac{R_{M}\left(c t_{a}\right)}{r}\right) A^{2}\left(c t_{a}, r\right)\left(d c t_{D}\right)^{2}+\frac{d r^{2}}{1-\frac{R_{M}\left(c t_{a}\right)}{r}}+r^{2}\left(d \theta^{2}+\sin ^{2} \theta d \phi^{2}\right)$,

where the integrability condition on the coordinate $c t_{D}$ constrains the temporal scale factor $A$, requiring that it satisfies

$$
\frac{\partial}{\partial r} \frac{1}{A}=-\frac{\partial}{\partial c t_{a}} \frac{\Delta}{A}
$$

If $\ddot{R}_{M}=0$, a solution can be demonstrated for the coordinate transformation. The reduced coordinate $\zeta$ will be defined by

$$
\zeta\left(c t_{a}, r\right) \equiv \frac{R_{M}\left(c t_{a}\right)}{r}
$$

The temporal scale factor $A$ is assumed to approach unity for vanishing $\dot{R}_{M}$, giving the usual static Schwarzschild coordinates. The coefficient $A(\zeta)$ then satisfies

$$
\zeta \frac{\partial}{\partial \zeta} \frac{1}{A(\zeta)}=\dot{R}_{M} \frac{\partial}{\partial \zeta} \frac{\Delta(\zeta)}{A(\zeta)} \quad, \quad A(\zeta)=\exp \int_{\zeta_{o}}^{\zeta}\left[\frac{\frac{\partial \Delta(\tilde{\zeta})}{\partial \tilde{\zeta}} \dot{R}_{M} d \tilde{\zeta}}{\Delta(\tilde{\zeta}) \dot{R}_{M}-\tilde{\zeta}}\right]
$$

where $\zeta_{o}$ is the reduced coordinate of correspondence with the Schwarzschild metric. The behavior of this function is shown in Fig. 1, The temporal scale factor is seen to behave in a manner of interest for several values of $\zeta$ in the figure. One of the zeroes of the temporal scale factor $A\left(\zeta_{A}\right)=0$ corresponds to an anomaly in the diagonal time coordinate $c t_{D}$, while the singular behavior in $A$ near $\zeta \sim 1$ corresponds to the coordinate anomaly in $c t_{a}$ associated with the radial mass scale $R_{M}$. The zero in the temporal scale factor for $\zeta=0$ dis-allows the use of $r \rightarrow \infty$ for correspondence of $c t_{D}$ with the temporal coordinate of asymptotic Schwarzschild geometry.

The form of the diagonalized time can be integrated from the analyticity requirement associated with the coordinate transformation. Suppose that the form of this coordinate is given by

$$
c t_{D}\left(c t_{a}, r\right)=R_{M}\left(c t_{a}\right) F(\zeta) .
$$




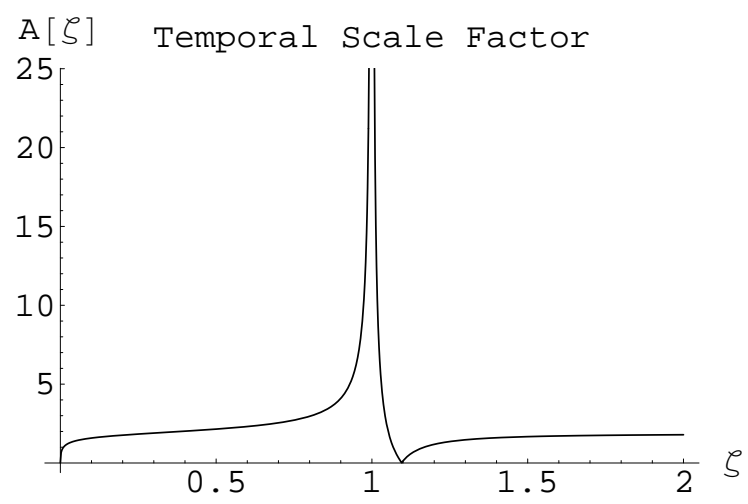

Figure 1: Scale Factor for an evaporating black hole with $\dot{R}_{M}=0.1$

Substitution into Eq. 2.5 gives conditions

$$
\zeta^{2} F^{\prime}(\zeta)=\frac{\Delta(\zeta)}{A(\zeta)} \quad, \quad \dot{R}_{M}\left[F(\zeta)+\zeta F^{\prime}(\zeta)\right]=\frac{1}{A(\zeta)}
$$

which can immediately be solved for the function $F(\zeta)$ to give

$$
c t_{D}\left(c t_{a}, r\right)=\left(\frac{\dot{R}_{M} \sqrt{\zeta}-\zeta(1-\zeta)}{\zeta(1-\zeta) A(\zeta)}\right) \frac{R_{M}\left(c t_{a}\right)}{\dot{R}_{M}} .
$$

This diagonal form will be useful in the development of the conformal coordinates needed to construct the Penrose diagram for this space-time.

\subsection{Construction of conformal coordinates}

Conformal coordinates are convenient for examining the causal structure of the space-time. The conformal coordinates $\left(c t_{*}, r_{*}\right)$ for this spacetime can be obtained from the diagonal coordinates using the transformation

$$
\begin{gathered}
B\left(d c t_{*}-D d r_{*}\right) \equiv A \sqrt{1-\zeta} d c t_{D} \\
B\left(-D d c t_{*}+d r_{*}\right) \equiv \frac{d r}{\sqrt{1-\zeta}} .
\end{gathered}
$$

The metric then takes the form

$$
d s^{2}=B^{2}\left(1-D^{2}\right)\left[-\left(d c t_{*}\right)^{2}+d r_{*}^{2}\right]+r^{2}\left(d \theta^{2}+\sin ^{2} \theta d \phi^{2}\right) .
$$


It is convenient to define scaling parameters $W_{ \pm}$using the forms

$$
\begin{aligned}
B & \equiv \frac{1}{2} \frac{1}{\sqrt{1-\zeta}} \frac{W_{+}+W_{-}}{W_{+} W_{-}} \\
D & \equiv \frac{W_{+}-W_{-}}{W_{+}+W_{-}} .
\end{aligned}
$$

The metric can then be re-written in terms of re-scaled parameters $\tilde{W}_{ \pm}$ defined by

$$
\tilde{W}_{ \pm} \equiv(1 \mp \sqrt{\zeta}) W_{ \pm}
$$

in the form

$$
d s^{2}=\frac{-\left(d c t_{*}\right)^{2}+d r_{*}^{2}}{\tilde{W}_{+} \tilde{W}_{-}}+r^{2}\left(d \theta^{2}+\sin ^{2} \theta d \phi^{2}\right) .
$$

Defining conformal light cone coordinates $d u_{ \pm} \equiv d c t_{*} \pm d r_{*}$, the transformation Eq. 2.14 requires that

$$
d u_{ \pm}=\left[(1 \pm \sqrt{\zeta}) d c t_{a} \pm d r\right] \tilde{W}_{ \pm}(\zeta)
$$

The functions $\tilde{W}_{ \pm}$then must satisfy the integrability conditions for the conformal coordinates

$$
-\zeta \frac{d}{d \zeta}\left[(1 \pm \sqrt{\zeta}) \tilde{W}_{ \pm}\right]= \pm \dot{R}_{M} \frac{d}{d \zeta} \tilde{W}_{ \pm}
$$

This equation can be re-written in a form that more directly demonstrates the singular points in the equation

$$
\frac{d}{d \zeta}\left[\left\{\zeta(1 \pm \sqrt{\zeta}) \pm \dot{R}_{M}\right\} \tilde{W}_{ \pm}\right]=(1 \pm \sqrt{\zeta}) \tilde{W}_{ \pm}
$$

The roots $\zeta_{S}$ of the quantity in the brackets $\left\{\zeta_{S}\left(1 \pm \sqrt{\zeta_{S}}\right) \pm \dot{R}_{M}\right\}=0$ represent light-like surfaces, one of which corresponds to the horizon. The solutions for the re-scaled factors $\tilde{W}_{ \pm}$are of the form

$$
\tilde{W}_{ \pm}(\zeta)=\frac{ \pm \dot{R}_{M}}{\left\{\zeta(1 \pm \sqrt{\zeta}) \pm \dot{R}_{M}\right\}} \exp \left[\int_{0}^{\zeta} \frac{\left(1 \pm \sqrt{\zeta^{\prime}}\right) d \zeta^{\prime}}{\left\{\zeta^{\prime}\left(1 \pm \sqrt{\zeta^{\prime}}\right) \pm \dot{R}_{M}\right\}}\right]
$$



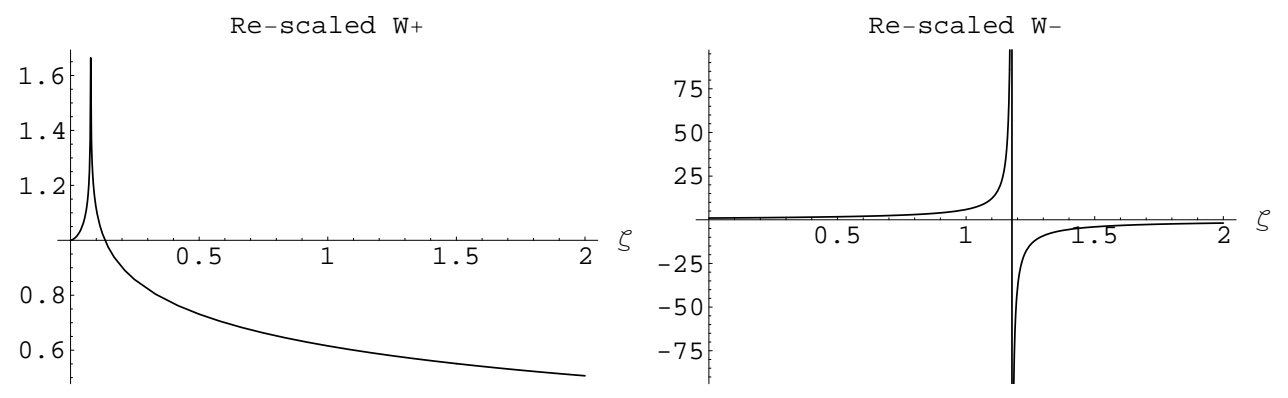

Figure 2: Re-scaled transformation factors $\tilde{W}_{ \pm}(\zeta)$ for $\dot{R}_{M}=0.1$

An analytic form for this solution can be obtained, and an example numerical solution is demonstrated in Fig. 2. The singular behavior in $\tilde{W}_{-}$ corresponds to the horizon, while that in $\tilde{W}_{+}$corresponds to an incoming light-like surface.

Utilizing the same technique used to derive Eq. 2.13, the forms of the conformal coordinates can be integrated to obtain

$$
\begin{aligned}
& c t_{*}=\frac{r}{2}\left[\left\{1+\frac{\zeta(1+\sqrt{\zeta})}{\dot{R}_{M}}\right\} \tilde{W}_{+}(\zeta)+\left\{-1+\frac{\zeta(1-\sqrt{\zeta})}{\dot{R}_{M}}\right\} \tilde{W}_{-}(\zeta)\right] \\
& r_{*}=\frac{r}{2}\left[\left\{1+\frac{\zeta(1+\sqrt{\zeta})}{\dot{R}_{M}}\right\} \tilde{W}_{+}(\zeta)-\left\{-1+\frac{\zeta(1-\sqrt{\zeta})}{\dot{R}_{M}}\right\} \tilde{W}_{-}(\zeta)\right]
\end{aligned}
$$

These equations provide the transformation between the conformal coordinates $\left(c t_{*}, r_{*}\right)$ and the non-orthogonal coordinates $\left(c t_{a}, r\right)$.

\subsection{Penrose diagram of the evaporating black hole}

In a Penrose diagram, the space-time structure is represented using functions of the conformal coordinates in Eq. 2.23 (with light-like surfaces always represented by lines with slope \pm 1 ) and the entire space-time mapped onto a finite diagram. Since light-like surfaces are represented in a simple way, causal relationships can be determined in such diagrams in a straightforward manner. Each point $\left(c t_{a}, r\right)$ in the Penrose diagram developed here will represent the surface of a sphere of area $4 \pi r^{2}$ at distant observer time $t_{a}$. 


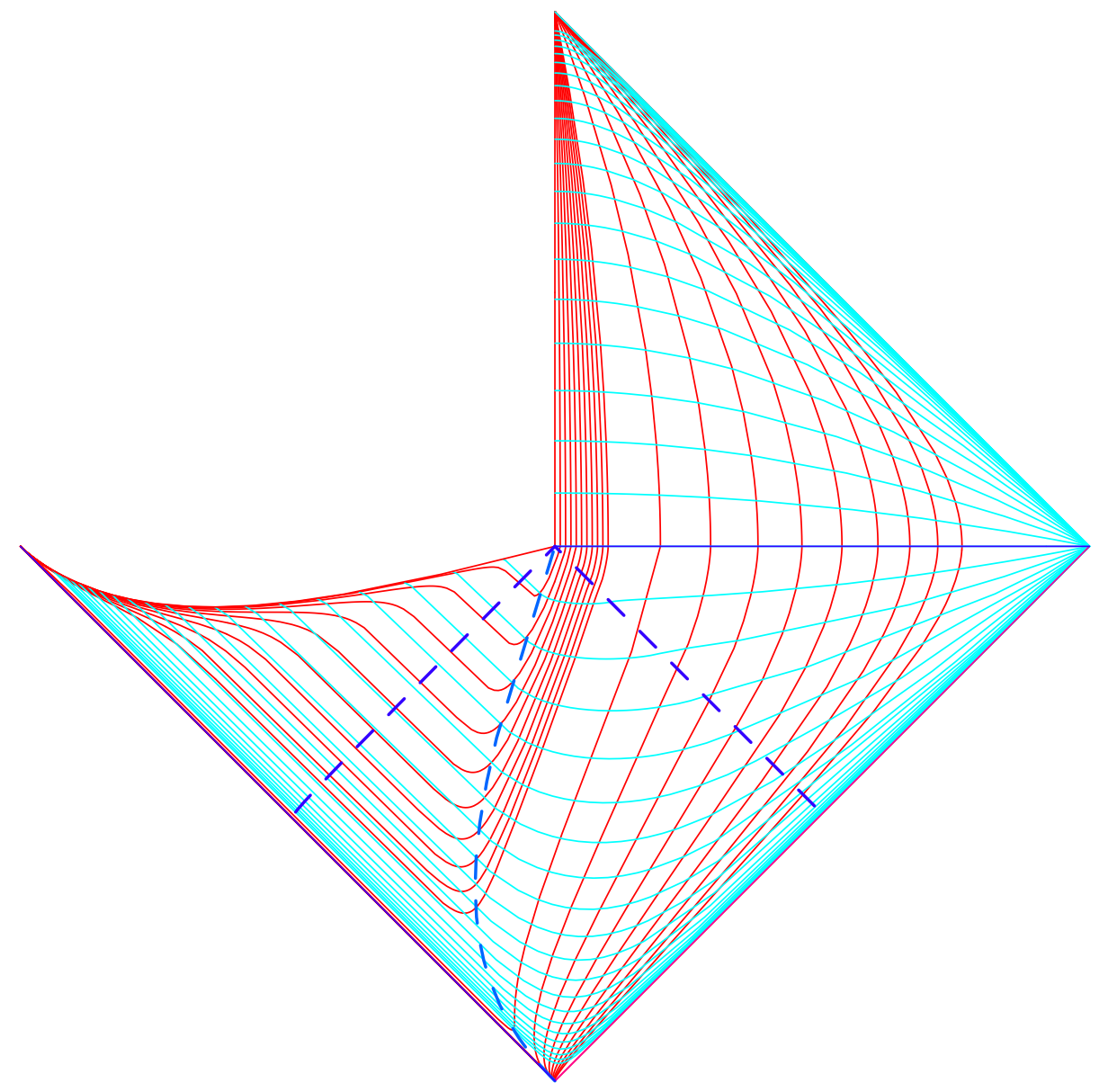

Figure 3: Penrose diagram for black hole that evaporates steadily until it reaches zero mass at $t_{a}=0$ 
The Penrose diagram in Figure 3 demonstrates the expected global structure of this spherically symmetric black hole that evaporates at a steady rate of change in the radial mass scale $r=R_{M}\left(c t_{a}\right)$ with respect to the distant observer's time coordinate $c t_{a}$. In the distant past, the area of the horizon of the black hole is indefinitely large, $R_{H} \rightarrow \infty$ as $t_{a} \rightarrow-\infty$. The diagram uses hyperbolic tangents of a scaled multiple of the conformal coordinates in Eq. 2.23] for the construction. In the diagram, the red curves that are time-like in the right hand regions represent curves of constant $r$, originally graded from $r=0$ in tenths, then in multiples of the chosen scale. The curves of constant radial coordinate $r$ all originate at the far left corner of the diagram representing $t_{a}=-\infty$ and terminate at the uppermost corner representing $t_{a}=\infty$. The green curves that are space-like in the right hand regions represent curves of constant $c t_{a}$ graded in multiples of the given scale. All constant $c t_{a}$ curves originate on the curve $r=0$ and terminate at the far right corner of the diagram representing $r=\infty$. The $c t_{a}=$ constant and $r=$ constant curves, although not orthogonal, do serve as valid coordinates, each intersecting at only one point on the diagram.

The diagram has several regions and features of interest. The right triangular region in the upper right region of the diagram bounded by the red line $r=0$ on the left, the solid blue line $c t_{a}=0$ on the bottom, and $r=\infty, c t_{a}=\infty$ as the hypotenuse, corresponds to the Minkowski spacetime after the completion of the (coherent) evaporation at $t_{a}=0$. For $t_{a}<0$ the red curve $r=0$ becomes space-like and serves as the topmost bound of the interior region of the black hole (the bottom left side of the diagram), taking the curved form demonstrated between $t_{a}=-\infty$ and $t_{a}=0$. In the space-time region with significant curvature, the curve $r=0$ tracks a physical singularity with a strength related to the shrinking mass scale. The horizon is a light-like surface representing the outermost set of out-going photons that will eventually hit the fading singularity. On the Penrose diagram, the horizon is represented by the dashed blue outgoing light-like line just beneath the physical singularity $r=0$ in the interior. The horizon also represents the singular curve in the re-scaled transformation factor $\hat{W}_{-}$. The incoming light-like surface represented by the dashed blue line originating from the lower right region of the diagram is the singular curve in the re-scaled transformation factor $\hat{W}_{+}$. The evolving radial mass scale $R_{M}\left(c t_{a}\right)$ is represented by the dashed blue curve originating at the bottom corner of the diagram. Each of these dashed curves terminates 
at $\left(c t_{a}=0, r=0\right)$ when the evaporation ceases, and cross indefinitely larger radial coordinates in the distant past. The radial mass scale $R_{M}$ associated with the $\left(c t_{a}, r\right)$ coordinate anomaly in the highly curved metric of the black hole geometry is clearly seen to differ from the horizon coordinate $R_{H}$. Indeed, $R_{M}\left(c t_{a}\right)$ cannot be a light-like surface, since outgoing photons at this coordinate will momentarily be stationary, while $R_{M}$ itself is steadily decreasing. The behaviors of the coordinate lines are clearly anomalous in the vicinity of the radial mass scale $r \leq R_{M}$. However, there is no physical singularity introduced at this coordinate anomaly.

From the diagram, one can immediately determine that no photon emitted from the region interior to the horizon $0<r<R_{H}$ can escape hitting the physical singularity $r=0$. Similarly, as discussed in section 2.2. an outgoing photon emitted from the region $R_{H}<r \leq R_{M}$ is seen to escape hitting the singularity. No incoming photon emitted from the region to the right of the singular curve of the re-scaled transformation factor $\hat{W}_{+}$(the incoming dashed blue line) can hit the physical singularity or communicate with the interior region. The horizon is always seen to lie within the radial mass scale $R_{H}<R_{M}$ as expected for the evaporating black hole. The physical singularity $r=0$, the horizon $R_{H}$ (which is proportional to the radial mass scale), and the radial mass scale $R_{M}$ are seen to vanish together, leaving a time-like curve $r=0$ associated with the final no curvature Minkowski space-time, represented as the upper triangular region in the diagram subsequent to complete evaporation of the singularity.

\section{Conclusions}

The large-scale geometry of a black hole of steadily decreasing mass scale has demonstrated interesting aspects. One might expect that a black hole that has a horizon of infinite area as the time used by a distant observer tends to $-\infty$ would eventually contain any given observer within that horizon at an early enough time. The diagram in Fig. 3 clearly demonstrates that a substantial region of space-time lies outside of the regions of significant curvature, even outside the region of causal in-going exchange with the interior region of the black hole. This region can be parameterized in terms of multiples of the shrinking mass scale, always remaining external to the interior regions. The diagram also directly demonstrates the distinction between the horizon and the radial mass scale for this dynamic 
geometry.

The diagram demonstrates some intriguing aspects of "Asymptopia". As shown in Eq. 2.10, the zero at $\zeta=0$ in the temporal scale factor $A(\zeta)$ that transforms the original coordinate $c t_{a}$ to the diagonal "Schwarzschildlike" time $c t_{D}$ at $r \rightarrow \infty$ dis-allows the use of the asymptotic Schwarzschild geometry for correspondence of the Schwarzschild temporal parameter with the diagonal time $c t_{D}$. This means that some aspects of the asymptotic space-time described using the coordinates $\left(c t_{D}, r\right)$ that corresponds to Schwarzschild space-time at fixed $\zeta_{o}$ are not shared by corresponding asymptotic aspects of that particular Schwarzschild space-time. This additionally manifests by the observation that for static geometries, the form of $c t_{D}$ is ill-defined[2] in the asymptotic regime.

The "radiations" associated with the evaporation of this black hole are of a spatially coherent nature, vanishing on the space-like volume $c t_{a}=0$. Similar coherence is naturally found in the quantum nature of interactions. The dynamics for scalar quantum fields in this geometry is being examined, and will be presented in a future submission. In addition, the authors are examining diagrams describing systems that begin an accretion process and end a subsequent evaporation process using modifications of the results presented here. One should be able to gain insight into the life cycle of a black hole from these explorations.

\section{Acknowledgments}

BAB would like to acknowledge the support of the NASA Administrator's Fellowship Program.

\section{References}

[1] "Coordinates with non-singular curvature for a time-dependent black hole horizon", J. Lindesay, gr-qc/0609019 (2006), Foundations of Physics online DOI 10.1007/s10701-007-9146-4, 15 May 2007, 16 pages, .

[2] "The river model of black holes", A.J.S. Hamilton and J.P. Lisle, gr-qc/0411060 (2004) 14 pages. 
[3] "Production and decay of evolving horizons", A.B. Nielsen and M. Visser, gr-qc/0510083 (2006) 25 pages, Classical and Quantum Gravity 23 (2006) 4637-4658. 